\title{
Analysis of Flexible Batch Service Queueing System to Constrict Waiting Time of Customers
}

\author{
Deena Merit C. K. (D) and Haridass $M$ (iD \\ Department of Mathematics, PSG College of Technology, Coimbatore 641 004, Tamil Nadu, India \\ Correspondence should be addressed to Deena Merit C. K.; ckd.maths@psgtech.ac.in
}

Received 18 July 2021; Accepted 29 November 2021; Published 30 December 2021

Academic Editor: Mijia Yang

Copyright (c) 2021 Deena Merit C. K. and Haridass M. This is an open access article distributed under the Creative Commons Attribution License, which permits unrestricted use, distribution, and reproduction in any medium, provided the original work is properly cited.

\begin{abstract}
When the required number of customers is available in the general bulk service (GBS) queueing system, the server begins service. Otherwise, the server will remain inactive until the number of consumers in the queue reaches that minimum required number. Customers that have already come must wait throughout this time, regardless of their arrival time. In some circumstances, like specimens awaiting testing in a clinical laboratory or perishable commodities awaiting delivery, it is necessary to finish services before the expiration date. It might only be achievable if consumers' waiting times are kept under control. As a result, the flexible general bulk service (FGBS) rule is developed in this article to provide flexibility in batching. The effectiveness of FGBS implementation has been demonstrated using two examples: a clinical laboratory and a distribution center. To justify the suggested model, a simulation study and numerical illustration are provided.
\end{abstract}

\section{Introduction}

The server in a typical queueing system provides services to a single customer or a batch of customers. In terms of waiting time, the batch service is always deemed to be superior to the single service. Furthermore, the number of customers served in batch service is always greater than the number of consumers provided in single service. The batch size can be any number, but selecting a suitable batch size is essential, since it influences the customer's waiting time. The batching strategy with the least amount of waiting time and the lowest cost is regarded the best. As a result, selecting a suitable batching strategy is critical.

General bulk service (GBS) rule performs better among many batching techniques because the batch size depends upon the length of the queue. GBS rule was first introduced by Neuts [1]. In that, both the minimum and maximum limits, referred to as $a$ and $b$, determine the batch size. The server begins to provide service only when at least $a$ units are present in the queue. Immediately after completion of the first service, all the existing customers will be taken for service when the number of customers waiting in the queue is greater than or equal to $a$ but less than or equal to $b$. When the number of customers exceeds $b$, the first $b$ customers are taken for service. But the next batch will be taken for service only after the queue size reaches $a$ in case the customers are lesser than the minimum batch capacity $a$. The server and the existing customers have to wait until the number of customers reaches minimum batch size. This leads to an increase in the average waiting time of a customer and reduces server utilization.

Allowing the server to work on a subsidiary job or taking a break might boost server utilization. Vacation, in general, refers to the unavailability of the server to provide a service. Several authors studied various combinations of bulk arrival general bulk service and vacation queueing systems. A survey on server vacation has been analyzed by Doshi [2]. Sasikala and Indhira [3] did a detailed survey on bulk service queueing models. Only a few of the authors listed below took actions to decrease the average waiting time of the customer by modifying the general bulk service rule.

Balasubramanian et al. [4] explored a bulk service queueing system with overloading and multiple vacations. If the server finds that there are more than $N$ customers after 
completing a service or vacation, the server will increase its service capacity, known as overload, and serve $N$ customers in a batch. Bulk queue with modified vacation policy was investigated by Jeyakumar and Arumuganathan [5]. In that, if the queue size is less than $a$ after $M$ vacations, the server remains idle until the queue size reaches the minimum threshold value $a$. Following that, Balasubramanian and Arumuganathan [6] enhanced that with state-dependent arrival.

A bulk arrival bulk service queueing system with working vacation was studied by Jeyakumar and Senthilnathan [7]. Instead of being idle during the vacation period, the server will work at a different rate while on working vacation. Arumuganathan and Jeyakumar [8] examined an $M^{X} / G(a, c, b) / 1$ queueing system in which the server can choose between a single service and a bulk service based on the number of consumers in the queue while entering into service. Only when $a$ customers are in queue will the service begin. When the queue length is greater than $a$, the server will select a single service and serve it one at a time. When the queue length is $c,(c \geq a)$, the server will pick bulk service and serve at most $b$ consumers in a batch. Deepa and Azhagappan [9] looked at batch arrival single and bulk service queues with multiple vacation closedown and repairs. If the queue size is less than $a$, the server provides single service; if the queue size is greater than $a$, the server provides bulk service. If there are no customers in line at the completion epoch of any service, the server will begin a closedown and thereafter enter multiple vacations. All of the foregoing work was done in order to reduce the average waiting time of the customer. In all these mentioned cases, the batch size was modified in the GBS rule concerning only the number of customers accumulated in the queue.

Batching based only on the number of customers accumulating in the queue has several drawbacks. When the client is perishable, for example, the customer may rot before the service is completed due to a time delay in batching. As a result, in such cases, the GBS rule must be modified. This article introduces the flexible general bulk service (FGBS) rule, which modifies the GBS rule regarding the average waiting time of a consumer in the queue. In FGBS, the server starts bulk service when (i) there are at least $a$ customers in the queue or (ii) there are less than $a$ customers in the queue but the measured average waiting time of these customers in the queue is greater than or equal to the waiting time tolerance $T$. The server becomes idle if the number of customers at the completion epoch of service is less than the minimum batch size $a$ and the average waiting time of a customer in the queue is less than time $T$.

The FGBS rule has been introduced to ensure that customers who are waiting in the queue due to batching inadequacy get served promptly. The FGBS rule uses the average waiting time of the inadequate number of customers in the queue rather than individual waiting time, since mean is the best central tendency value that reflects a group. Individual waiting times, such as the waiting time of the customer at the front of the line, might result in very tiny batches and raise the cost.
Additional possibilities for accelerating the general bulk service include increasing the number of servers, increasing the service rate, and lowering the minimum batch size. Increasing the rate of service in some queueing systems, such as processing user requests at a base station or using an elevator for transit, may not be possible. Increasing the number of servers and lowering the minimum batch size will raise the cost, which is unnecessary when traffic is low; yet, FGBS performs well under these circumstances.

In the present era, computer is a part in almost all queue management systems. Queue Management Software (QMS) is used in such queues to track entries, exits, the average waiting time of all customers in queue $W_{q}$, and so on. The waiting time tolerance $T$ can be calculated using the experience, the expiration period, or the customer's direct/ indirect feedback. If the number of customers in the queue is insufficient for bulk service but the average waiting time of the accumulated customers $W q$ in the queue exceeds the waiting time tolerance $T$, customise the QMS to send an alert or notification to provide bulk service to the waiting customers after the ongoing service is completed.

Prior to implementation, it is always a good idea to run a simulation analysis. Through multiple iterations, simulation analysis can be utilised to determine an appropriate waiting time tolerance $T$ for a given situation. So validation and verification can be performed in a virtual environment and initial implementation challenges in a real-time context can be avoided. As a result, simulation is employed throughout this research to demonstrate the value of the proposed concept FGBS.

In this paper, the FGBS rule is applied to a multiple vacation $M^{X} / G(a, b) / 1$ queueing system. The assumptions for GBS and FGBS with vacation are given in Table 1. To pick the optimum value of $T$, the flexible general bulk service rule is compared to the general bulk service rule. In addition, the suggested flexible general bulk service rule is tested in two scenarios, namely, a clinical laboratory and a distribution center, to determine its efficacy.

The rest of the article is organised as follows: The model description and notations used in the model are found in Section 2. In Section 3, the model's probability generating function is derived. Section 4 describes the simulation of the proposed model and the determination of the optimal value of $T$. The motivation for the suggested model is discussed in Section 5. In this section, the FGBS rule is applied to manage clinical laboratories queueing system and queue of perishable items waiting for delivery. In Section 6, conclusion and some future extensions are mentioned.

\section{Model Description}

The following are the assumptions made for the multiple vacation $\mathrm{MX} / G(a, b) / 1$ queueing system with FGBS rule.

Arrival of batches of customers follows the Poisson process with composite arrival rate $\lambda$. Actual number of customers in any arriving module is a random variable $X$ with probability distribution $\operatorname{Pr}(X=k)=g_{k}$. Single server provides bulk service to the customers in the FCFS discipline. Service time follows a general distribution with rate $\mu$. 
TABLE 1: Service rule and vacation policy.

\begin{tabular}{lcccc}
\hline Service rule & \multicolumn{2}{c}{ Number of customers waiting in queue } & Vacation policy \\
\hline & $N<a$ & $a \leq N<b$ & $N>b$ & $N<a$ \\
GBS rule & No service & Bulk of $N$ & Bulk of $b$ & $N<a$ and $W_{q}<T$ \\
FGBS rule & Bulk of $N$ if $W_{q} \geq T$ & Bulk of $N$ & Bulk of $b$ & \\
\hline
\end{tabular}

The server will start to provide service for the customers only when at least $a$ units are present in the queue or else average waiting time of a customer in queue $W_{q}$ is greater than or equal to the waiting time tolerance $T$. Maximum service capacity is $b,(b \geq a)$. After completion of a service or a vacation,

(i) the number of customers in the waiting line is less than or equal to $b$ and greater than or equal to $a$, then all the existing customers are taken for service;

(ii) more than $b$ number of customers are in the queue, then the first $b$ customers are taken into service;

(iii) the number of customers is lesser than the minimum batch capacity $a$ and $W_{q}<T$, then the server leaves for vacation, which follows an exponential distribution with rate $v$;

(iv) the number of customers is lesser than the minimum batch capacity $a$ but $W_{q} \geq T$, then the entire queue is taken for service.

The server takes multiple vacations until at the end of any vacation either the queue size greater than or equal to $a$ or $W_{q} \geq T$.

2.1. Notations. The following are the notations used in this model: $\lambda$ is arrival rate of the customers; $\mu$ is service rate of the server; $v$ is vacation rate; $X$ is group size random variable of arrival; $g_{k}=\operatorname{Pr}(X=k)-\mathrm{PMF}$ of $X ; S$ is random variable for service time; $W_{q}$ is random variable for average waiting time of a customer accumulated in the queue; $S(\cdot)$ is cumulative distribution function of service time; $V($.$) is cu-$ mulative distribution function of vacation time; $S^{0}(t)$ is remaining service time at any time $t ; V^{0}(t)$ is remaining vacation time at any time $t ; s(x)$ is density function of $S ; v(x)$ is density function of $V ; f\left(w_{q}\right)$ is density function of $W_{q}$.

\section{System Size Distribution}

Steady-state system size distribution is obtained in this section. Various states of the system at time $t$ are defined as follows:

$$
\text { Define } \begin{aligned}
N_{q}(t) & =\text { number of customers in the queue at any time } t, \\
N_{s}(t) & =\text { number of customers in the service at any time } t \text { and, } \\
Y(t) & = \begin{cases}0, & \text { server is on vacation, } \\
1, & \text { server is busy, }\end{cases} \\
Z(t) & =j, \text { the server is on } j^{\text {th }} \text { vacation. }
\end{aligned}
$$

The state probabilities are defined as

$$
\begin{aligned}
P_{i, j}(x, t) \mathrm{d} t=\operatorname{Pr}\left\{N_{s}(t)=i, N_{q}(t)=j, x<S^{0}(t)<x+\mathrm{d} t, Y(t)=1\right\}, & a \leq i \leq b, j>0, \\
Q_{n, j}(x, t) \mathrm{d} t=\operatorname{Pr}\left\{N_{q}(t)=n, x<V^{0}(t)<x+\mathrm{d} t, Y(t)=0, Z(t)=j\right\}, & n>0, j>1 .
\end{aligned}
$$

3.1. System State Equations. All possible system states are identified and corresponding equations are written for an infinitesimal $\Delta t$ using supplementary variable technique. For example, $P_{i, 0}(t+\Delta t)$, that is, the probability that the state with $i,(1 \leq i \leq a-1)$ number of customers in the system and the queue is empty at time $(t+\Delta t)$, is obtained from the following possible events:

(i) Server is providing bulk service for $i$ customers, the queue is empty at time $t$, and no one arrives in $\Delta t$. (ii) Average waiting time of customers in the queue exceeds $T$ at the completion epoch of bulk of $m$ at $t$, and hence service starts in $\Delta t$.

(iii) Average waiting time of customers in the queue exceeds $T$ at the completion epoch of any vacation at $t$, and hence service starts in $\Delta t$.

$$
\begin{aligned}
P_{i, 0}(x-\Delta t, t+\Delta t)= & P_{i, 0}(x, t)(1-\lambda \Delta t)+\sum_{m=1}^{b} P_{m, i}(0, t) \int_{T}^{\infty} f\left(w_{q}\right) \mathrm{d} w_{q} s(x) \Delta t \\
& +\sum_{l=1}^{\infty} Q_{l, i}(0, t) \int_{T}^{\infty} f\left(w_{q}\right) \mathrm{d} w_{q} s(x) \Delta t, \quad 1 \leq i \leq a-1 .
\end{aligned}
$$


Similarly, the following equations are obtained:

$$
\begin{aligned}
P_{i, 0}(x-\Delta t, t+\Delta t) & =P_{i, 0}(x, t)(1-\lambda \Delta t)+\sum_{m=1}^{b} P_{m, i}(0, t) s(x) \Delta t+\sum_{l=1}^{\infty} Q_{l, i}(0, t) s(x) \Delta t, \quad a \leq i \leq b, \\
P_{i, j}(x-\Delta t, t+\Delta t) & =P_{i, j}(x, t)(1-\lambda \Delta t)+\sum_{k=1}^{j} P_{i, j-k}(x, t) \lambda g_{k} \Delta t, \quad 1 \leq i \leq b-1, j \geq 1, \\
P_{b, j}(x-\Delta t, t+\Delta t) & =P_{b, j}(x, t)(1-\lambda \Delta t)+\sum_{m=1}^{b} P_{m, b+j}(0, t) s(x) \Delta t+\sum_{k=1}^{j} P_{b, j-k}(x, t) \lambda g_{k} \Delta t+\sum_{l=1}^{\infty} Q_{l, b+j}(0, t) s(x) \Delta t, \quad j \geq 1, \\
Q_{1,0}(x-\Delta t, t+\Delta t) & =Q_{1,0}(x, t)(1-\lambda \Delta t)+\sum_{m=1}^{b} P_{m, 0}(0, t) v(x) \Delta t, \\
Q_{1, n}(x-\Delta t, t+\Delta t) & =Q_{1, n}(x, t)(1-\lambda \Delta t)+\sum_{m=1}^{b} P_{m, n}(0, t) v(x) \Delta t+\sum_{k=1}^{n} Q_{1, n-k}(x, t) \lambda g_{k} \Delta t, \quad n \geq 1, \\
Q_{j, 0}(x-\Delta t, t+\Delta t) & =Q_{j, 0}(x, t)(1-\lambda \Delta t)+Q_{j-1,0}(0, t) v(x) \Delta t, \quad j \geq 2, \\
Q_{j, n}(x-\Delta t, t+\Delta t) & =Q_{j, n}(x, t)(1-\lambda \Delta t)+Q_{j-1, n}(0, t) \int_{0}^{T} f\left(w_{q}\right) \mathrm{d} w_{q} v(x) \Delta t+\sum_{k=1}^{n} Q_{j, n-k}(x, t) \lambda g_{k} \Delta t, \quad j \geq 2, n \geq 1 .
\end{aligned}
$$

In the steady state, the transient effects get faded. Hence, the following steady-state equations are obtained using the assumptions $P_{i, j}(x, t)=P_{i, j}(x)$ and $Q_{n, j}(x, t)=Q_{n, j}(x)$ in the above system of equations:

$$
\begin{gathered}
-\frac{\mathrm{d}}{\mathrm{d} x} P_{i, 0}(x)=-\lambda P_{i, 0}(x)+\sum_{m=1}^{b} P_{m, i}(0) \int_{T}^{\infty} f(w) \mathrm{d} w s(x)+\sum_{l=1}^{\infty} Q_{l, i}(0) \int_{T}^{\infty} f(w) \mathrm{d} w s(x), \quad 1 \leq i \leq a-1, j=0 \\
-\frac{\mathrm{d}}{\mathrm{d} x} P_{i, 0}(x)=-\lambda P_{i, 0}(x)+\sum_{m=1}^{b} P_{m, i}(0) s(x)+\sum_{l=1}^{\infty} Q_{l, i}(0) s(x), a \leq i \leq b-1, \\
-\frac{\mathrm{d}}{\mathrm{d} x} P_{i, j}(x)=-\lambda P_{i, j}(x)+\sum_{k=1}^{j} P_{i, j-k}(x) \lambda g_{k}, a \leq i \leq b-1, j \geq 1 \\
\mathrm{~d} x P_{b, j}(x)=-\lambda P_{b, j}(x)+\sum_{m=1}^{b} P_{m, b+j}(0) s(x)+\sum_{k=1}^{j} P_{b, j-k}(x) \lambda g_{k} t+\sum_{l=1}^{\infty} Q_{l, b+j}(0) s(x), \quad j \geq 1 \\
-\frac{\mathrm{d}}{\mathrm{d} x} Q_{1,0}(x)=-\lambda Q_{1,0}(x)+\sum_{m=1}^{b} P_{m, 0}(0) v(x) \\
-\frac{\mathrm{d}}{\mathrm{d} x} Q_{1, n}(x)=-\lambda Q_{1, n}(x)+\sum_{m=1}^{b} P_{m, n}(0) v(x)+\sum_{k=1}^{n} Q_{1, n-k}(x) \lambda g_{k}, n \geq 1, \\
-\frac{\mathrm{d}}{\mathrm{d} x} Q_{j, 0}(x)=-\lambda Q_{j, 0}(x)+Q_{j-1,0}(0) v(x), j \geq 2 \\
-\lambda Q_{j, n}(x)+Q_{j-1, n}(0) \int_{0}^{T} f(w) \mathrm{d} w v(x)+\sum_{k=1}^{n} Q_{j, n-k}(x) \lambda g_{k}, \quad j \geq 2, n \geq 1 .
\end{gathered}
$$


The Laplace-Stieltjes transform is defined as follows:

$$
\begin{aligned}
\widetilde{P}_{i, n}(\theta) & =\int_{0}^{\infty} e^{-\theta x} P_{i, n}(x) \mathrm{d} x, \\
\widetilde{Q}_{j, n}(\theta) & =\int_{0}^{\infty} e^{-\theta x} Q_{j, n}(x) \mathrm{d} x .
\end{aligned}
$$

Taking Laplace-Stieltjes transform on both sides of (5) to (12),

$$
\begin{aligned}
& \theta \widetilde{P}_{i, 0}(\theta)-P_{i, 0}(0)=\lambda \widetilde{P}_{i, 0}(\theta)-\sum_{m=1}^{b} P_{m, i}(0) \int_{T}^{\infty} f(w) \mathrm{d} w \widetilde{S}(\theta)-\sum_{l=1}^{\infty} Q_{l, i}(0) \int_{T}^{\infty} f(w) \mathrm{d} w \widetilde{S}(\theta), \quad 1 \leq i \leq a-1, j=0 \\
& \theta \widetilde{P}_{i, 0}(\theta)-P_{i, 0}(0)=\lambda \widetilde{P}_{i, 0}(\theta)-\sum_{m=1}^{b} P_{m, i}(0) \widetilde{S}(\theta)-\sum_{l=1}^{\infty} Q_{l, i}(0) \widetilde{S}(\theta), \quad a \leq i \leq b-1, \\
& \theta \widetilde{P}_{i, j}(\theta)-P_{i, j}(0)=\lambda \widetilde{P}_{i, j}(\theta)-\sum_{k=1}^{j} \widetilde{P}_{i, j-k}(\theta) \lambda g_{k}, \quad a \leq i \leq b-1, j \geq 1, \\
& \theta \widetilde{P}_{b, j}(\theta)-P_{b, j}(0)=\lambda \widetilde{P}_{b, j}(0)-\sum_{m=1}^{b} P_{m, b+j}(0) \widetilde{S}(\theta)-\sum_{k=1}^{j} \widetilde{P}_{b, j-k}(\theta) \lambda g_{k} t-\sum_{l=1}^{\infty} Q_{l, b+j}(0) \widetilde{S}(\theta), \quad j \geq 1, \\
& \theta \widetilde{Q}_{1,0}(\theta)-Q_{1,0}(0)=\lambda \widetilde{Q}_{1,0}(\theta)-\sum_{m=1}^{b} P_{m, 0}(0) \widetilde{V}(\theta), \\
& \theta \widetilde{Q}_{1, n}(\theta)-Q_{1,0}(0)=\lambda \widetilde{Q}_{1, n}(\theta)-\sum_{m=1}^{b} P_{m, n}(0) \widetilde{V}(\theta)-\sum_{k=1}^{n} \widetilde{Q}_{1, n-k}(\theta) \lambda g_{k}, \quad n \geq 1 \\
& \theta \widetilde{Q}_{j, 0}(\theta)-Q_{j, 0}(0)=\lambda \widetilde{Q}_{j, 0}(\theta)-Q_{j-1,0}(0) \tilde{V}(\theta), \quad j \geq 2, \\
& \theta \widetilde{Q}_{j, n}(\theta)-Q_{j, n}(0)=\lambda \widetilde{Q}_{j, n}(\theta)-Q_{j-1, n}(0) \int_{0}^{T} f(w) \mathrm{d} w \widetilde{V}(\theta)-\sum_{k=1}^{n} \widetilde{Q}_{j, n-k}(\theta) \lambda g_{k}, \quad j \geq 2, n \geq 1 .
\end{aligned}
$$

The following marginal/partial probability generating functions are defined to obtain the probability generating function (PGF) of the system size at an arbitrary time:

$$
\begin{aligned}
\widetilde{P}_{i}(z, \theta) & =\sum_{j=0}^{\infty} \widetilde{P}_{i, j}(\theta) z^{j}, \\
P_{i}(z, 0) & =\sum_{j=0}^{\infty} P_{i, j}(0) z^{n}, \quad 1 \leq i \leq b, \\
\widetilde{Q}_{j}(z, \theta) & =\sum_{n=0}^{\infty} \widetilde{Q}_{j, n}(\theta) z^{n}, \\
Q_{j}(z, 0) & =\sum_{n=0}^{\infty} Q_{j, n}(0) z^{n}, \quad j \geq 1 .
\end{aligned}
$$

Multiplying (18) and (19) by $z^{0}$ and $z^{n}, n=1,2, \ldots a-1$, respectively, we get 


$$
[\theta-\lambda+\lambda X(z)] \widetilde{Q}_{1}(z, \theta)=Q_{1}(z, 0)-\tilde{V}(\theta) \int_{0}^{T} f(w) \mathrm{d} w\left[\sum_{n=0}^{a-1} \sum_{m=1}^{b} P_{m n}(0) z^{n}\right]
$$

Multiplying (20) and (21) by $z^{0}$ and $z^{n}, n=1,2, \ldots a-1$, respectively, we get

$$
[\theta-\lambda+\lambda X(z)] \widetilde{Q}_{j}(z, \theta)=Q_{j}(z, 0)-\tilde{V}(\theta) \sum_{n=0}^{a-1} Q_{j-1}(0) z^{n} \int_{0}^{T} f(w) \mathrm{d} w, \quad j \geq 2 .
$$

Multiplying (14) and (16) by $z^{0}$ and $z^{j}, j=1,2, \ldots a-1$, respectively, we get

$$
[\theta-\lambda+\lambda X(z)] \widetilde{P}_{i}(z, \theta)=P_{i}(z, 0)-\widetilde{S}(\theta) \int_{0}^{T} f(w) \mathrm{d} w\left[\sum_{m=1}^{b} P_{m, i}(0)+\sum_{l=1}^{\infty} Q_{l, i}(0)\right], \quad 0 \leq i \leq a-1
$$

Multiplying (15) and (16) by $z^{0}$ and $z^{j}$, $j=a, a+1, \ldots b-1$, respectively, we get

$$
[\theta-\lambda+\lambda X(z)] \widetilde{P}_{i}(z, \theta)=P_{i}(z, 0)-\widetilde{S}(\theta)\left[\sum_{m=1}^{b} P_{m, i}(0)+\sum_{l=1}^{\infty} Q_{l, i}(0)\right], \quad a \leq i \leq b-1
$$

Multiplying (15) and (17) by $z^{0}$ and $z^{j}$, we get

$$
[\theta-\lambda+\lambda X(z)] \widetilde{P}_{b}(z, \theta)=P_{b}(z, 0)-\widetilde{S}(\theta)\left[\sum_{j=0}^{\infty} \sum_{m=1}^{b} P_{m, b+j}(0) z^{j}+\sum_{j=0}^{\infty} \sum_{l=1}^{\infty} Q_{l, b+j}(0) z^{j}\right]
$$

Or

$$
z^{b}[\theta-\lambda+\lambda X(z)] \widetilde{P}_{b}(z, \theta)=z^{b} P_{b}(z, 0)-\widetilde{S}(\theta)\left[\sum_{m=1}^{b} P_{m}(z, 0)-\sum_{j=0}^{b-1} \sum_{m=1}^{b} P_{m, j}(0) z^{j}+\sum_{l=1}^{\infty} Q_{l}(z, 0)-\sum_{j=0}^{b-1} \sum_{l=1}^{\infty} Q_{l, j}(0) z^{j}\right]
$$


When $\theta=\lambda-\lambda X(z)$, the above equations gives

$$
\begin{aligned}
& Q_{1}(z, 0)=\tilde{V}(\lambda-\lambda X(z)) \int_{0}^{T} f(w) \mathrm{d} w\left[\sum_{n=0}^{a-1} \sum_{m=1}^{b} P_{m n}(0) z^{n}\right] \\
& Q_{j}(z, 0)=\tilde{V}(\lambda-\lambda X(z)) \sum_{n=0}^{a-1} Q_{j-1}(0) z^{n} \int_{0}^{T} f(w) \mathrm{d} w, \quad j \geq 2, \\
& P_{i}(z, 0)=\widetilde{S}(\lambda-\lambda X(z)) \int_{0}^{T} f(w) \mathrm{d} w\left[\sum_{m=1}^{b} P_{m, i}(0)+\sum_{l=1}^{\infty} Q_{l, i}(0)\right], \quad 0 \leq i \leq a-1, \\
& P_{i}(z, 0)=\widetilde{S}(\lambda-\lambda X(z))\left[\sum_{m=1}^{b} P_{m, i}(0)+\sum_{l=1}^{\infty} Q_{l, i}(0)\right], \quad a \leq i \leq b-1, \\
& z^{b} P_{b}(z, 0)=\widetilde{S}(\lambda-\lambda X(z))\left[\sum_{m=1}^{b} P_{m}(z, 0)-\sum_{j=0}^{b-1} \sum_{m=1}^{b} P_{m, j}(0) z^{j}+\sum_{l=1}^{\infty} Q_{l}(z, 0)-\sum_{j=0}^{b-1} \sum_{l=1}^{\infty} Q_{l, j}(0) z^{j}\right] a \leq i \leq b-1 \\
& =\widetilde{S}(\lambda-\lambda X(z))\left[\sum_{m=1}^{b-1} P_{m}(z, 0)+P_{b}(z, 0)-\sum_{j=0}^{b-1} \sum_{m=1}^{b} P_{m, j}(0) z^{j}+\sum_{l=1}^{\infty} Q_{l}(z, 0)-\sum_{j=0}^{b-1} \sum_{l=1}^{\infty} Q_{l, j}(0) z^{j}\right], \\
& {\left[z^{b}-\widetilde{S}(\lambda-\lambda X(z))\right] P_{b}(z, 0)=\widetilde{S}(\lambda-\lambda X(z))\left[\sum_{m=1}^{b-1} P_{m}(z, 0)-\sum_{j=0}^{b-1} \sum_{m=1}^{b} P_{m, j}(0) z^{j}+\sum_{l=1}^{\infty} Q_{l}(z, 0)-\sum_{j=0}^{b-1} \sum_{l=1}^{\infty} Q_{l, j}(0) z^{j}\right],} \\
& \widetilde{Q}_{1}(z, \theta)=\frac{[\tilde{V}(\lambda-\lambda X(z))-\tilde{V}(\theta)]}{[\theta-\lambda+\lambda X(z)]} \int_{0}^{T} f(w) \mathrm{d} w\left[\sum_{n=0}^{a-1} \sum_{m=1}^{b} P_{m n}(0) z^{n}\right] \\
& \widetilde{Q}_{j}(z, \theta)=\frac{[\widetilde{V}(\lambda-\lambda X(z))-\widetilde{V}(\theta)]}{[\theta-\lambda+\lambda X(z)]} \sum_{n=0}^{a-1} Q_{j-1}(0) z^{n} \int_{0}^{T} f(w) \mathrm{d} w, \quad j \geq 2, \\
& \widetilde{P}_{i}(z, \theta)=\frac{[\widetilde{S}(\lambda-\lambda X(z))-\widetilde{S}(\theta)]}{[\theta-\lambda+\lambda X(z)]} \int_{0}^{T} f(w) \mathrm{d} w\left[\sum_{m=1}^{b} P_{m, i}(0)+\sum_{l=1}^{\infty} Q_{l, i}(0)\right] 0 \leq i \leq a-1, \\
& \widetilde{P}_{i}(z, \theta)=\frac{[\widetilde{S}(\lambda-\lambda X(z))-\widetilde{S}(\theta)]}{[\theta-\lambda+\lambda X(z)]}\left[\sum_{m=1}^{b} P_{m, i}(0)+\sum_{l=1}^{\infty} Q_{l, i}(0)\right], \quad a \leq i \leq b-1, \\
& \widetilde{P}_{b}(z, \theta)=\frac{[\widetilde{S}(\lambda-\lambda X(z))-\widetilde{S}(\theta)]}{[\theta-\lambda+\lambda X(z)]\left[z^{b}-\widetilde{S}(\lambda-\lambda X(z))\right]}\left[\sum_{m=1}^{b-1} P_{m}(z, 0)-\sum_{j=0}^{b-1} \sum_{m=1}^{b} P_{m, j}(0) z^{j}+\sum_{l=1}^{\infty} Q_{l}(z, 0)-\sum_{j=0}^{b-1} \sum_{l=1}^{\infty} Q_{l, j}(0) z^{j}\right] .
\end{aligned}
$$


Let $P(z)$ be the probability generating function of the queue size at an arbitrary time epoch. Then

$$
\begin{aligned}
P(z) & =\sum_{i=1}^{a-1} \widetilde{P}_{i}(z, 0)+\sum_{i=a}^{b-1} \widetilde{P}_{i}(z, 0)+\widetilde{P}_{b}(z, 0)+\sum_{j=1}^{\infty} \widetilde{Q}_{j}(z, 0) \\
& =\sum_{i=1}^{a-1} \frac{[\widetilde{S}(\lambda-\lambda X(z))-1]}{[-\lambda+\lambda X(z)]} \int_{0}^{T} f(w) \mathrm{d} w\left[\sum_{m=1}^{b} P_{m, i}(0)+\sum_{l=1}^{\infty} Q_{l, i}(0)\right]+\sum_{i=a}^{b-1} \frac{[\widetilde{S}(\lambda-\lambda X(z))-1]}{[-\lambda+\lambda X(z)]}\left[\sum_{m=1}^{b} P_{m, i}(0)+\sum_{l=1}^{\infty} Q_{l, i}(0)\right] \\
& +\frac{[\widetilde{S}(\lambda-\lambda X(z))-\widetilde{S}(\theta)]}{[\theta-\lambda+\lambda X(z)]\left[z^{b}-\widetilde{S}(\lambda-\lambda X(z))\right]}\left[\sum_{m=1}^{b-1} P_{m}(z, 0)-\sum_{j=0}^{b-1} \sum_{m=1}^{b} P_{m, j}(0) z^{j}+\sum_{l=1}^{\infty} Q_{l}(z, 0)-\sum_{j=0}^{b-1} \sum_{l=1}^{\infty} Q_{l, j}(0) z^{j}\right] \\
& +\frac{[\widetilde{V}(\lambda-\lambda X(z))-1]}{[-\lambda+\lambda X(z)]} \int_{0}^{T} f(w) \mathrm{d} w\left[\sum_{n=0}^{a-1} \sum_{m=1}^{b} P_{m n}(0) z^{n}\right]+\sum_{j=2}^{\infty} \frac{[\widetilde{V}(\lambda-\lambda X(z))-1]}{[-\lambda+\lambda X(z)]} \sum_{n=0}^{a-1} Q_{j-1}(0) z^{n} \int_{0}^{T} f(w) \mathrm{d} w .
\end{aligned}
$$

Let $P_{i}=\sum_{m=1}^{b} P_{m i}(0), q_{i}=\sum_{l=1}^{\infty} Q_{l i}(0)$, and $c_{i}=p_{i}+q_{i}$.

$$
\left.P(z)=[\widetilde{S}(\lambda-\lambda X(z))-1]\left[z^{b}\left[\int_{T}^{\infty} f(w) \mathrm{d} w \sum_{i=1}^{a-1} c_{i}+\sum_{i=a}^{b-1} c_{i}\right]-\sum_{i=1}^{b-1} c_{i} z^{i}\right]\right]+\frac{\left[z^{b}-1\right] \int_{0}^{T} f(w) \mathrm{d} w[\tilde{V}(\lambda-\lambda X(z))-1] \sum_{i=1}^{a-1} c_{i} z^{i}}{[\lambda+\lambda X(z)]\left[z^{b}-\widetilde{S}(\lambda-\lambda X(z))\right]}
$$

\section{Simulation Model}

The random variable $W q$ signifies the average waiting time of customers in the queue, whereas $f(w q)$ is the corresponding density function called average waiting time distribution. For the average waiting time distribution, no assumptions were used. Because obtaining performance measures of the suggested model via an analytical/closed form solution is tedious, simulation is used to justify the model. The transient and steady-state behaviours of a queueing system can be visualised using a simulation model. It can also provide performance measures that are not available in an analytical method, such as the number of customers who join the system, the number of customers who are served, and the average batch size. The suggested model's ARENA Document, as well as the descriptions used in the modules, is shown in Figure 1.

The simulation was conducted over the course of a year. Table 2 shows performance measures such as average number of customers and average waiting time of a customer in the queue under FGBS and GBS rules. In the table, the values assigned to both fixed and variable parameters are listed. The following are some observations:

(i) When the mean rate of arrival decreases, the interarrival time $1 / \lambda$ increases. As a result, the required number of customers for batching is slowly reached, resulting in a decrease in queue length and an increase in the average waiting time of a customer under GBS rule. The FGBS rule, on the other hand, controls the customer's waiting time.

(ii) When the mean service time $1 / \mu$ increases, the mean waiting time of a customer increases under both GBS and FGBS rules. However, the waiting time in the system with FGBS rule is lesser than that in the system with GBS rule.

(iii) Average waiting time of a customer grows when the minimum need for batch service $a$ increases in the system under the GBS rule. The FGBS rule works nicely in this case. It controls the number of customers waiting in the queue and the average waiting time of the customer in the queue.

(iv) The server's nonavailability increases as the vacation time $1 / v$ increases. The GBS rule increases the average customer waiting time, whereas the FGBS rule keeps it under control.

4.1. An Illustration to Find the Optimum Value of T. For a given set of parametric values, Table 2 displays the performance measures of a queue with GBS and FGBS rules. It was discovered that the FGBS rule efficiently controls the batching delay. In the case of the FGBS queue, $T$ is a factor that influences a customer's average waiting time; therefore it is critical to select appropriate $T$ for the model. Smaller $T$ value often makes small batches lead to an increase in cost. 


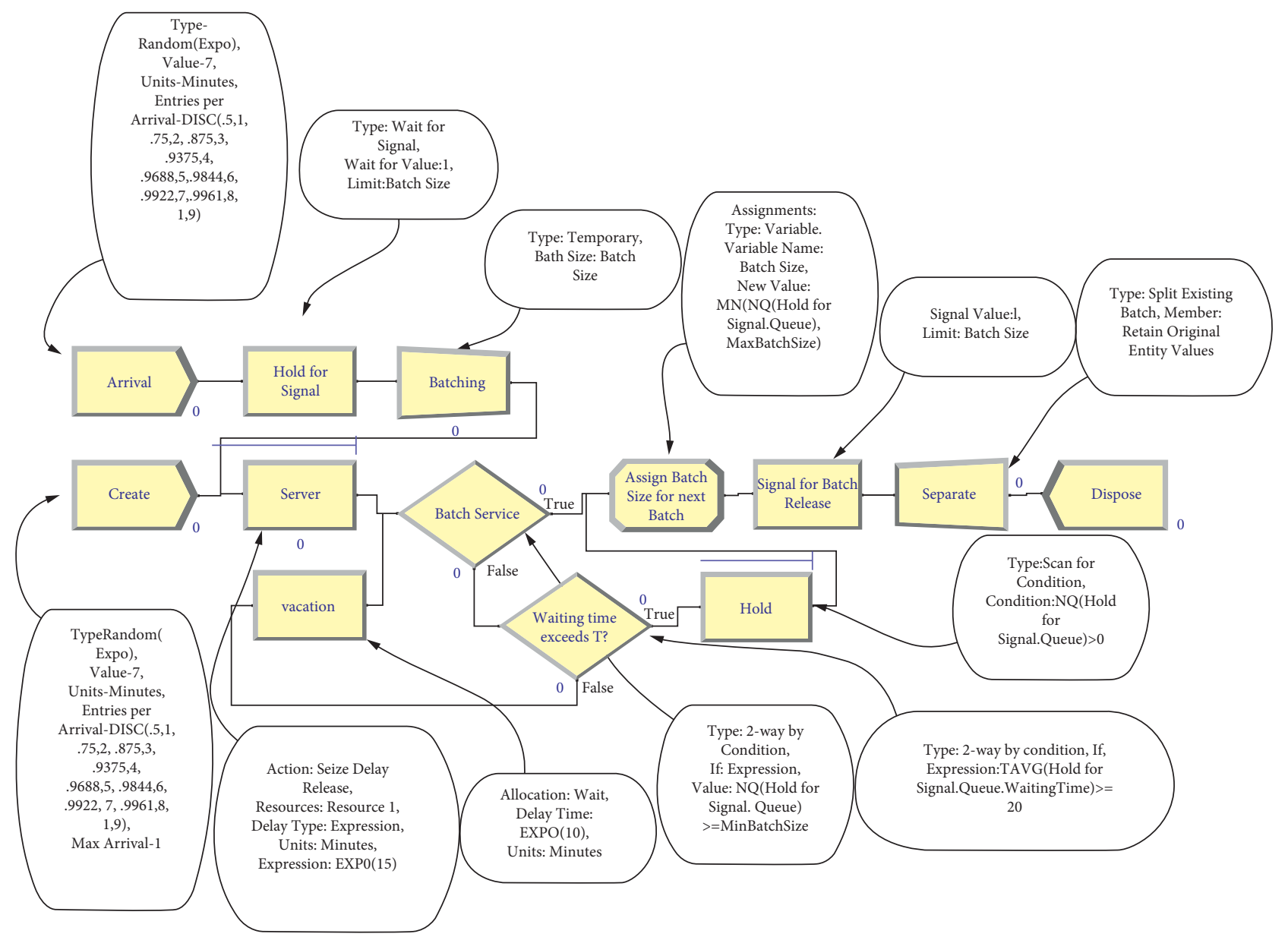

FIGURE 1: ARENA Document for the system with FGBS rule.

TABLE 2: Performance measures of FGBS and GBS queueing system.

\begin{tabular}{|c|c|c|c|c|c|c|}
\hline \multirow{2}{*}{$\begin{array}{l}\text { Fixed } \\
\text { Parameter value }\end{array}$} & \multirow{2}{*}{$\begin{array}{c}\text { Varying } \\
\text { Parameter }\end{array}$} & \multirow{2}{*}{$\begin{array}{l}\text { Varying parameter } \\
\text { Value }\end{array}$} & \multicolumn{2}{|c|}{ FGBS } & \multicolumn{2}{|c|}{ GBS } \\
\hline & & & $L_{q}$ & $W_{q}$ & $L_{q}$ & $W_{q}$ \\
\hline & & 7 & 5.5331 & 19.4289 & 5.9686 & 19.4500 \\
\hline$p=0.5, \mu=1 / 15$ & & 8 & 4.7795 & 19.5466 & 4.8454 & 19.6091 \\
\hline $1 / v=10, T=20$ & $1 / \lambda$ & 9 & 4.3072 & 19.6006 & 4.4027 & 19.8180 \\
\hline \multirow{3}{*}{$a=5, b=10$} & & 10 & 3.9214 & 19.7320 & 4.0033 & 20.1954 \\
\hline & & 11 & 3.5613 & 19.5977 & 3.7940 & 20.7675 \\
\hline & & 16 & 6.0795 & 21.4545 & 6.0146 & 21.4683 \\
\hline$p=0.5, \lambda=1 / 7$ & & 17 & 6.7644 & 23.6723 & 6.8101 & 24.0375 \\
\hline $1 / v=10, T=20$ & $1 / \mu$ & 18 & 7.0618 & 24.0544 & 6.9151 & 24.4400 \\
\hline \multirow[t]{3}{*}{$a=5, b=10$} & & 19 & 7.6609 & 27.4875 & 8.0874 & 28.7299 \\
\hline & & 20 & 9.6706 & 29.4958 & 8.3545 & 30.2270 \\
\hline & & 6 & 5.8598 & 20.4906 & .0017 & 21.1610 \\
\hline$p=0.5, \lambda=1 / 7$ & & 7 & 5.8292 & 20.5080 & 6.6472 & 23.4282 \\
\hline $1 / 7=10, T=20$ & $a$ & 8 & 5.8204 & 20.4608 & 7.3731 & 25.7064 \\
\hline \multirow[t]{3}{*}{$\mu=1 / 15, b=10$} & & 9 & 5.8762 & 20.5375 & 8.0629 & 28.7262 \\
\hline & & 10 & 5.8693 & 20.4320 & 8.6862 & 30.2426 \\
\hline & & 11 & 5.8927 & 20.4626 & 6.2302 & 21.8386 \\
\hline$p=0.5, \lambda=1 / 7$ & & 12 & 5.7161 & 20.0142 & 6.0551 & 21.6661 \\
\hline $1 / \mu=1 / 15, T=20$ & $1 / v$ & 13 & 5.9351 & 20.5204 & 6.5877 & 22.9786 \\
\hline \multirow[t]{2}{*}{$a=5, b=10$} & & 14 & 5.8690 & 20.4903 & 6.6665 & 23.3598 \\
\hline & & 15 & 5.8072 & 20.3694 & 6.5405 & 23.0509 \\
\hline
\end{tabular}


Setting a large $T$ value results in bigger batches, which may save costs but increases the average wait time of a customer. An optimal $T$ value, on the other hand, manages and minimises a customer's queue waiting time at the lowest possible cost. The $T$ value can be derived from the problem itself in some real-world situations like the blood samples awaiting clinical testing, perishable commodities awaiting delivery, and so on. If no such $T$ is available in the problem, one can use the ARENA Output Analyzer to get the optimum value of $T$. The procedure of choosing the correct $T$ value is given below.

Along with the FGBS simulation model in ARENA, a dat file is produced in the statistic module to collect the data of the variable BatchSize to identify the optimum $T$ value. Figure 2 depicts a spreadsheet view of the dat file. To find the optimum $T$ value, along with the FGBS simulation model in ARENA a .dat file is created in the statistic module to collect the statistics of the BatchSize. Spreadsheet view of the .dat file is shown in Figure 2.

For each $T$ value from 1 to 27 , a separate dat file was produced. These data files include data that is persistent across time. So, using the batch/truncate icon in Output Analyzer, a flt file was produced for each file. Using the Conf. Int (Mean) icon in the Output Analyzer, all of these $f t t$ files were uploaded to obtain the confidence interval of the average batch size. The results are shown in the graph of mean batch size with a confidence interval (95\%) in Figure 3 and a summary in Table 3 .

$p=0.5, \lambda=1 / 7, \mu=1 / 15, a=7, b=10,1 / v=10$.

The average waiting time of a customer in the queue under the GBS rule is 23.4282 for the parametric set of parameters $p=0.5,=1 / 7,=1 / 15, a=7, b=10,1 / v=10$ from Table 2. Figure 3 shows that when $T$ ranges from 1 to 11 , the average batch size does not change. Table 3 further shows that, for any $T$ between 1 and 11, the average batch size is 4.54 , the average waiting time of a customer is 18.1858 , and the total number of services is 4664 . Under the FGBS rule, a customer's minimal average waiting time is 18.1858 minutes. From $T=12$, the average batch size is beginning to rise. To identify an optimum $T$, the data in Table 3 are shown in Figure 4. From the graph, the average waiting time of a customer can be controlled from 18.185 8. As per the graph, the maximum $T$ value which minimises average waiting time of a customer in the queue, maximizes average batch size, and minimizes number of services is 18 . At the optimum $T$, the average waiting time of a customer is 18.660 5. As a result, if the $T$ value is not provided in the problem, the suitable $T$ value can be determined by prior experience or simulation based on the waiting time constraint.

\section{Application of Proposed Model}

Managing the customers having a fixed lifetime in a queue is a complicated task because it should not die or destroy before departing from the system. If it expires before the service completion the cost of product and service become a loss. Two common and frequently occurring situations are considered to show the effectiveness of the proposed model.
The first one is a clinical laboratory problem, where the specimens are alive only for a fixed lifetime after which they will expire. Analysis of the expired specimen creates a clinical error. The second one is the management of perishable items such as meat, vegetables, and flowers. The expiration of perishable items leads to wastage of the item itself.

\subsection{Flexible General Bulk Service in Clinical Laboratory.} Every day, clinical laboratories worldwide analyze billions of samples. Clinical laboratory results are used to provide essential information that allows reliable clinical decisionmaking for diagnosis, drug prescriptions, patient's admission, or discharge from the hospital. The complex process of laboratory testing finally provides a report, which is not error-free always. Clinical errors affect the emotional, financial, physical, and social behaviours of patients and sometimes technicians as well. Although Plebani and Carraro [10] demonstrated that $74 \%$ of laboratory errors did not affect patient's outcome, the other $26 \%$ becomes a patient care problem leading at least to further inappropriate investigations, discomfort, increased costs, even worse, improper care, modification to therapy, and so forth. Steps in clinical specimen testing are given in Figure 5. Lab testing process generally comprises three phases. The first is the preanalytical phase, which encompasses all the steps from the test request, sample collection, transport, and registration of the sample up to the start of specimen analysis. The second is the analytical phase, which involves the analysis of the samples and technical validation of the results. The third is the postanalytical phase, which includes the interpretation of the results, approval from the lab manager, and reporting to the physician. Laboratory errors might occur at any of these three phases.

The majority of diagnostic lab errors are either preanalytical (46-68.2\%) or postanalytical (18.5-47\%). Indeed, only $7-13 \%$ of errors occur during the analytical phase [11]. Detecting an error in the preanalytical phase is difficult and hardest to regulate and monitor because of the involvement of too many professionals like physicians, specialists of laboratory medicine, nurses, laboratory technicians, and phlebotomists [11]. Sakyi et al. [12] identified nine types of most common preanalytical errors. Delay in sample transportation is one of them. Nagat [13] investigated the frequency of preanalytical errors and identified that delay in sample transportation is a major cause for preanalytical laboratory error with a high frequency of 39\%. This delay time includes the time from the specimen collection to the start of the specimen analysis. It includes the delay caused by batching process and the blood samples are not reaching for the test on time. So, it is essential to minimize the delay in sample transportation to decrease the number of clinical errors that occur in the preanalytical phase.

5.1.1. Model Description in Clinical Laboratory. The clinical laboratory conducts blood test for both inpatients (IP) and outpatients (OP). For IP, the order of taking blood samples is prepared as a first step and then the ward nurses take the 


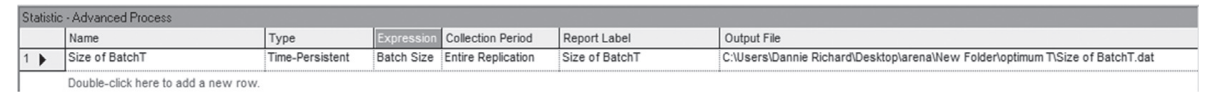

FIgURE 2: Spread sheet view of statistic module.

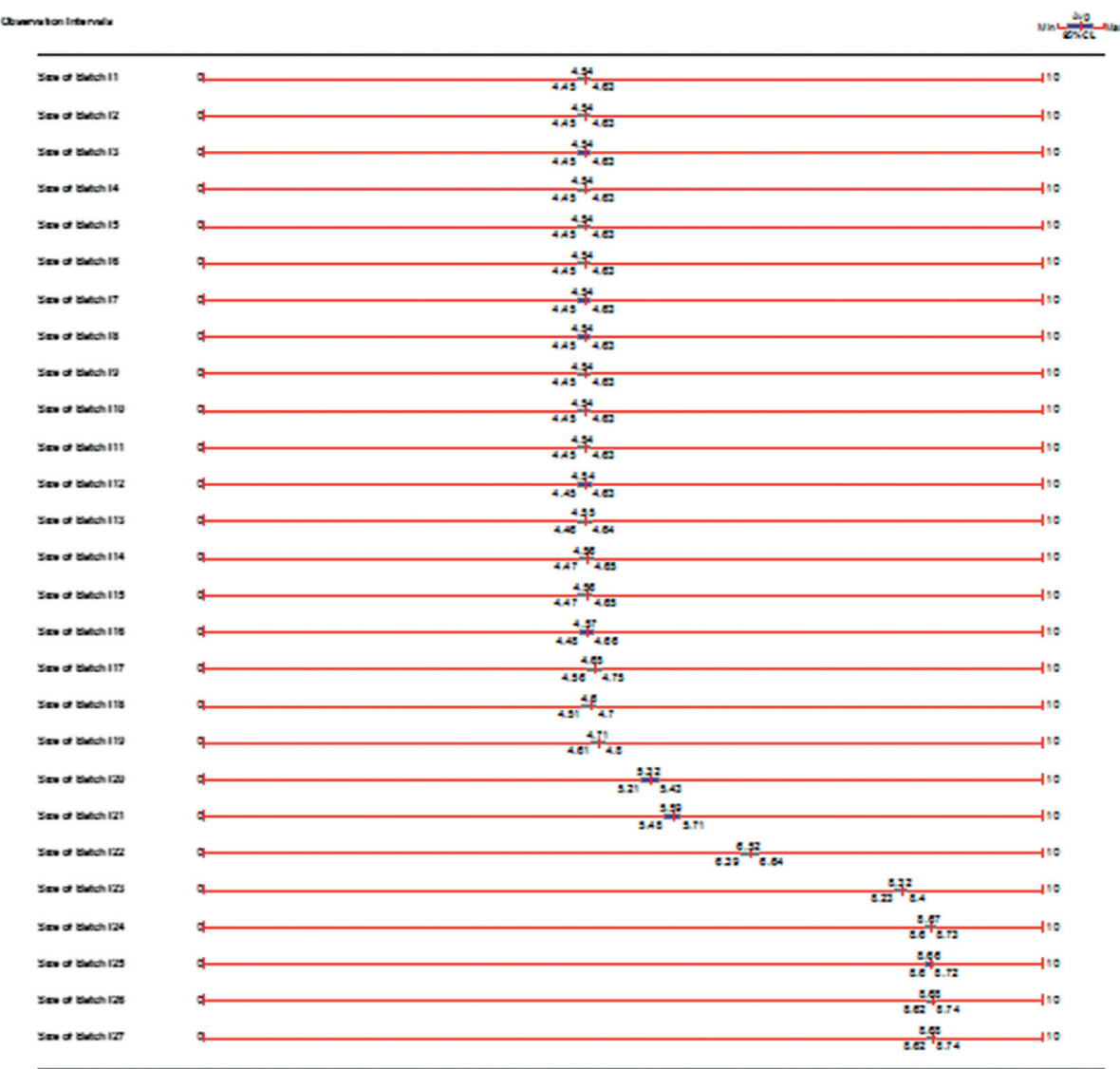

FIGURE 3: Confidence interval for mean batch size.

TABle 3: Performance measures for different $T$ value.

\begin{tabular}{|c|c|c|c|c|c|c|c|}
\hline$T$ & $\begin{array}{c}\text { Mean } \\
\text { Batch size }\end{array}$ & $W_{q}$ & $\begin{array}{l}\text { No. of } \\
\text { Services }\end{array}$ & $T$ & $\begin{array}{c}\text { Mean } \\
\text { Batch size }\end{array}$ & $W_{q}$ & $\begin{array}{c}\text { No. of } \\
\text { Services }\end{array}$ \\
\hline & & & (in thousands) & & & & (in thousands) \\
\hline 1 & 4.54 & 18.1858 & 4.664 & 15 & 4.56 & 18.3042 & 4.633 \\
\hline 2 & 4.54 & 18.1858 & 4.664 & 16 & 4.57 & 18.3535 & 4.621 \\
\hline 3 & 4.54 & 18.1858 & 4.664 & 17 & 4.65 & 18.387 & 4.503 \\
\hline 4 & 4.54 & 18.1858 & 4.664 & 18 & 4.6 & 18.6605 & 4.55 \\
\hline 5 & 4.54 & 18.1858 & 4.664 & 19 & 4.71 & 19.4133 & 4.386 \\
\hline 6 & 4.54 & 18.1858 & 4.664 & 20 & 5.32 & 20.508 & 3.578 \\
\hline 7 & 4.54 & 18.1858 & 4.664 & 21 & 5.59 & 21.3585 & 3.352 \\
\hline 8 & 4.54 & 18.1858 & 4.664 & 22 & 6.52 & 22.1731 & 2.719 \\
\hline 9 & 4.54 & 18.1858 & 4.664 & 23 & 8.32 & 22.8295 & 1.905 \\
\hline 10 & 4.54 & 18.1858 & 4.664 & 24 & 8.67 & 23.1117 & 1.796 \\
\hline 11 & 4.54 & 18.1858 & 4.664 & 25 & 8.66 & 22.8408 & 1.837 \\
\hline 12 & 4.54 & 18.2583 & 4.652 & 26 & 8.68 & 23.4282 & 1.787 \\
\hline 13 & 4.55 & 18.2863 & 4.651 & 27 & 8.68 & 23.4282 & 1.787 \\
\hline 14 & 4.56 & 18.2784 & 4.639 & 28 & 8.68 & 23.4282 & 1.787 \\
\hline
\end{tabular}

blood samples. If the number of samples is more than $a_{1}$, a nurse batches the collected samples with a maximum of $b_{1}$ samples and transports them to the laboratory. If the number of samples is inadequate, then the nurse goes on a vacation by taking a break or doing secondary work. The duration of vacation follows an exponential distribution 


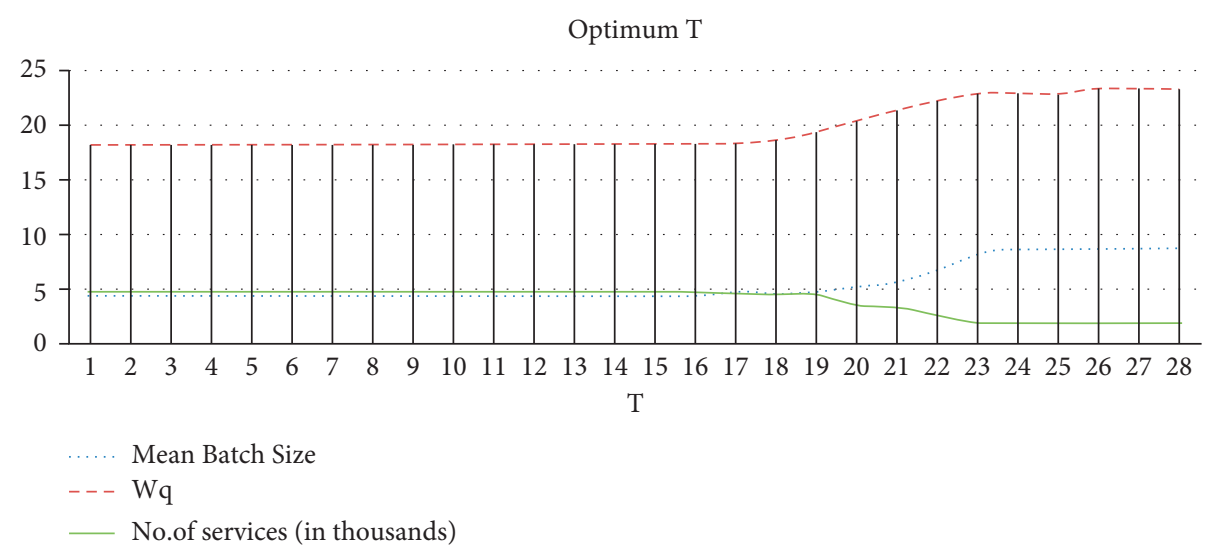

Figure 4: Performance measures with respect to $T$.

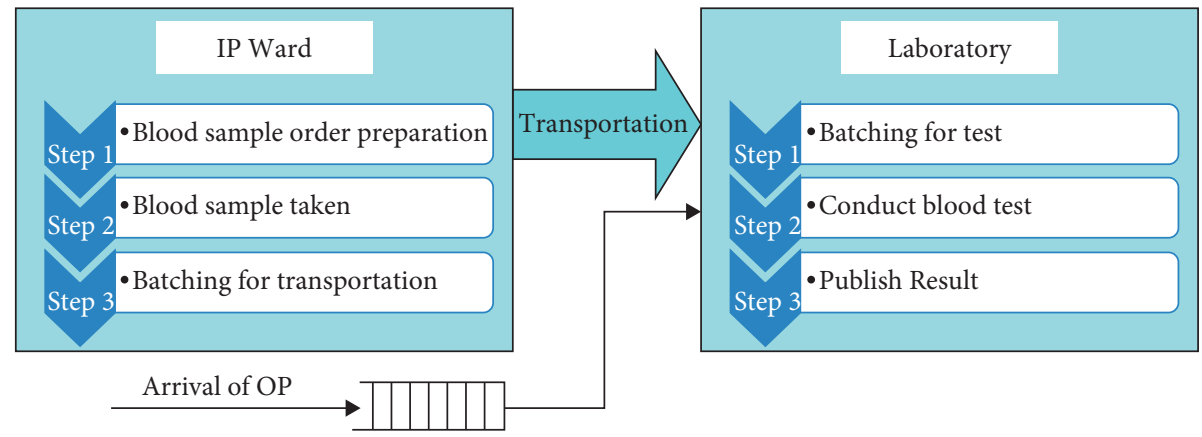

Figure 5: Clinical laboratory flow diagram.

with a rate of $v_{1}$. The blood samples of the OP are taken in the clinical laboratory itself. The collected samples are queued for the test bases on the FIFO queue discipline. The technician again batches the samples based on the capacity of the machine. The technician operates the machine only when at least $a_{2}$ samples are available and the machine can hold a maximum of $b_{2}$ samples. After running the machine, the technician publishes the result for diagnosis. A blood sample expires after a time frame $T$ because the blood structure changes. After that, it is not useful for medical analysis. The technician waits at least $a_{2}$ number of samples to optimize the operating cost of the testing machine, but the blood samples will expire in $T$ minutes. Therefore, the nurse in the ward and the technician in the clinical laboratory should not wait until the arrival of at least $a_{1}$ and $a_{2}$ numbers of samples, respectively. So, the batching rule of the nurse in the ward has to be modified as the specimens are taken for transport: either the availability of at least $a_{1}$ samples or the average waiting time of the blood samples in the ward exceeding $T_{1}$ minutes, whichever occurs first. Likewise, the batching of blood tests is done based on the first occurrence of either at least $a_{2}$ or average waiting time in the clinic not exceeding $T_{2}$ minutes. At completion epoch of service or vacation, if any of the conditions for batching fails to hold, then the nurse or the technician takes a vacation. The assumptions in Table 4 are used to simulate the model.
5.1.2. Numerical Illustration. The general bulk service (GBS) and flexible general bulk service (FGBS) rule models are simulated for the same set of values. The models ran for different values of minimum batch sizes $a_{1}$ and $a_{2}$ for 30 days. From that, the average waiting time of the customers in the queue, the total number of customers served at the end of the simulation run, the number of expiration of customers, and the cost of the models are tabulated in Tables 5 and 6 .

From Tables 5 and 6 , the deviation between the service rules GBS and FGBS on the performance measure of total number of customers served and cost of the system is not significant for different values of $a_{1}$ and $a_{2}$. But the aim of introducing the FGBS rule is to reduce the average waiting time of the customer and expiration. The performance measures like total number of expired customers and average waiting time of a customer in the system are significantly low. Hence, the FGBS satisfies its purpose. The graphs in Figures 6 and 7 illustrate this clearly.

Figures 6(a) and 6(b) show that the total number of expirations of customers is minimum under FGBS rule and considerably increases under GBS rule for higher values of $a_{1}$ and $a_{2}$, respectively.

Figures 7(a) and 7(b) show that the average waiting time of a customer in the system is in control under FGBS rule but it is out of control under GBS rule, especially at higher values of $a_{1}$ and $a_{2}$. 
TABLE 4: Assumptions.

\begin{tabular}{lcc}
\hline Parameter & Transport from ward & Testing machine \\
\hline Interarrival time & Exponential $\left(\lambda_{1}=1 / 7\right)$ & From ward + exponential $\left(\lambda_{2}=1 / 6\right)^{*}$ \\
Service time & Exponential $\left(\mu_{1}=1 / 15\right)$ & Exponential $\left(\mu_{2}=1 / 25\right)$ \\
Vacation time & Exponential $\left(v_{1}=1 / 10\right)$ & Exponential $\left(v_{2}=1 / 45\right)$ \\
Minimum batch size & $a_{1}=5$ & $a_{2}=24$ \\
Maximum batch size & $b_{1}=10$ & $b_{2}=32$ \\
Threshold & $T_{1}=20$ & $T_{2}=40$ \\
Cost-busy (hr) & 60 & 57 \\
Cost-idle (hr) & 60 & 57 \\
Cost per use & 20 & 300 \\
\hline
\end{tabular}

${ }^{*}$ Arrival of OP blood sample is scheduled from 9.00 am to $5 \mathrm{pm}$. Holding cost per entity is assumed as 10 .

TABle 5: Performance of the system with GBS and FGBS for different values of $a_{1}$.

\begin{tabular}{|c|c|c|c|c|c|c|c|c|}
\hline \multirow[b]{2}{*}{$a_{1}$} & \multicolumn{4}{|c|}{ General bulk service rule } & \multicolumn{4}{|c|}{ Flexible general bulk service rule } \\
\hline & $E(\mathrm{~W})$ & Served & Expired & Cost (Rs.) & $E(\mathrm{~W})$ & Served & Expired & Cost \\
\hline 2 & 107.2 & 8431 & 780 & 582345 & 54.68 & 8533 & 0 & 584643 \\
\hline 3 & 109.07 & 8577 & 792 & 588781 & 56.74 & 8567 & 9 & 568737 \\
\hline 4 & 110.11 & 8550 & 820 & 591135 & 58.89 & 8552 & 20 & 567272 \\
\hline 5 & 114.73 & 8562 & 1070 & 602047 & 60.36 & 8600 & 16 & 570181 \\
\hline 6 & 118.52 & 8422 & 1280 & 603946 & 60.14 & 8618 & 5 & 584641 \\
\hline 7 & 115.2 & 8488 & 1270 & 604616 & 60.64 & 8629 & 30 & 593734 \\
\hline 8 & 113.14 & 8508 & 1466 & 620084 & 60.08 & 8560 & 27 & 603234 \\
\hline 9 & 125.12 & 8599 & 1572 & 627462 & 60.23 & 8531 & 21 & 600620 \\
\hline
\end{tabular}

TABLE 6: Performance of the system with GBS and FGBS for different values of $a_{2}$.

\begin{tabular}{|c|c|c|c|c|c|c|c|c|}
\hline \multirow{2}{*}{$a_{2}$} & \multicolumn{4}{|c|}{ General bulk service rule } & \multicolumn{4}{|c|}{ Flexible general bulk service rule } \\
\hline & $E(\mathrm{~W})$ & No. served & Expired & Cost (Rs.) & $E(\mathrm{~W})$ & No. served & Expired & Cost \\
\hline 20 & 99.35 & 8533 & 559 & 563995 & 59.79 & 8601 & 29 & 566877 \\
\hline 21 & 108.17 & 8587 & 849 & 586365 & 60.01 & 8423 & 8 & 572454 \\
\hline 22 & 105.79 & 8505 & 810 & 580504 & 60.26 & 8650 & 33 & 577611 \\
\hline 23 & 112.79 & 8488 & 928 & 591951 & 60.97 & 8590 & 23 & 565880 \\
\hline 24 & 114.73 & 8562 & 1070 & 602047 & 60.36 & 8600 & 16 & 570181 \\
\hline 25 & 119.32 & 8438 & 1355 & 607912 & 60.1 & 8591 & 19 & 580819 \\
\hline 26 & 123.67 & 8474 & 1377 & 616739 & 60.01 & 8521 & 3 & 579136 \\
\hline 27 & 128.7 & 8413 & 1684 & 625369 & 60.08 & 8547 & 9 & 576013 \\
\hline
\end{tabular}

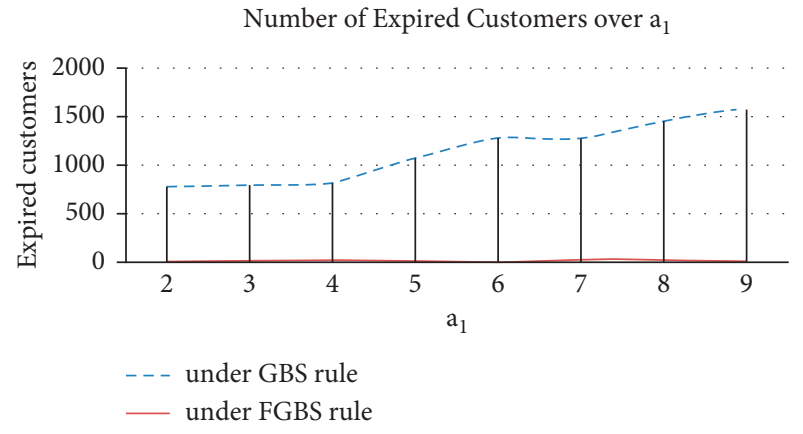

(a)

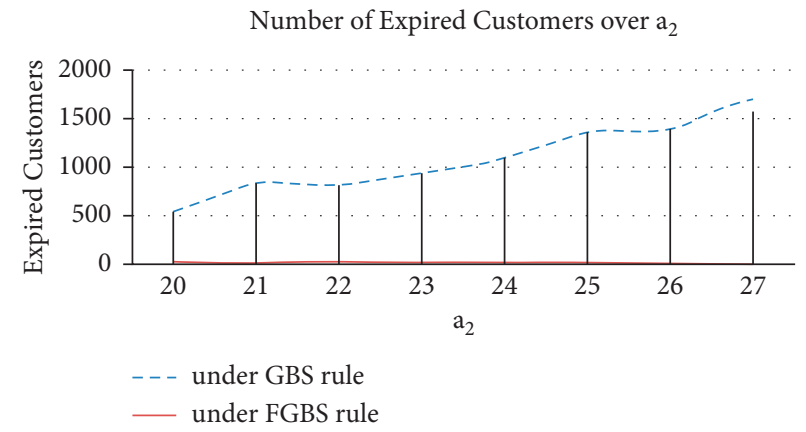

(b)

Figure 6: Expiration of customers.

5.2. Flexible General Bulk Service in Perishable Goods Management. The distribution center is designed to store the goods for retailers and wholesalers. The goods are distributed to another seller or customer directly from the distribution center. In today's world, these distribution centers are an important part of online retailers and e-commerce businesses. Distribution centers may store both perishable and nonperishable items. The fulfillment of the 


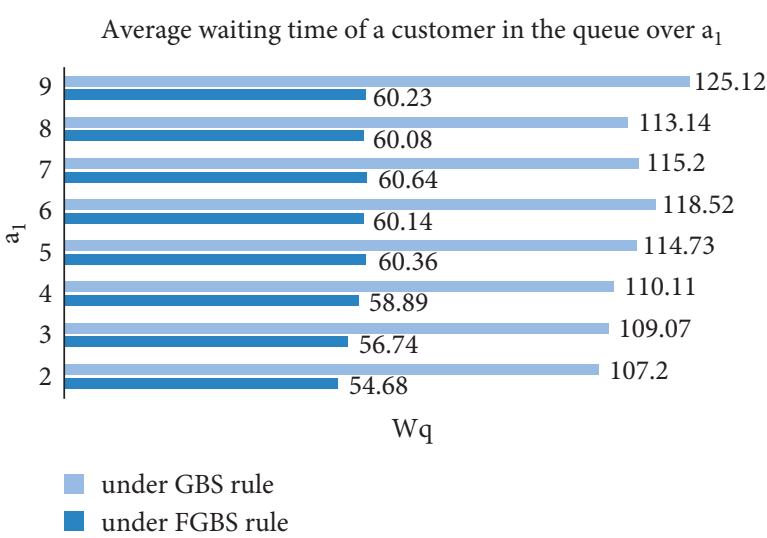

(a)

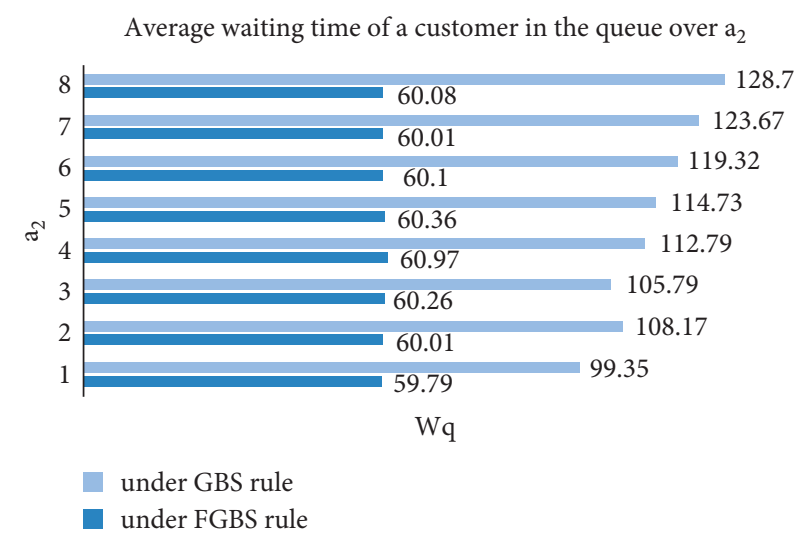

(b)

Figure 7: Average waiting time of a customer in the queue.

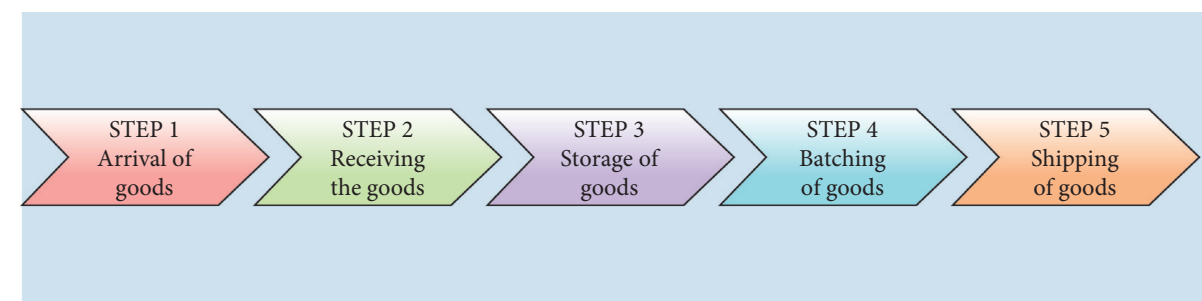

Figure 8: Stages in fulfillment of the order.

order has five steps as shown in Figure 8. There is a big difference between managing perishable goods and managing nonperishable goods. Storing and distributing the goods at low cost is the only goal of nonperishable items. Meanwhile, the time is the major constraint for perishable items because they have a limited lifespan after which they expire and become useless. Consider a distribution center where many suppliers are storing their perishable goods. There are less chances of delay until the third stage of order fulfillment. But usually delay happens in the fourth and fifth stage as they wait until at least $a$ units are available to reduce the shipping cost. This may minimize the transportation cost but sometimes the items may deteriorate before delivery. So it is essential to study storing and transporting the perishable items with optimum cost and minimum expiration. In this article, the distribution of perishable items is analyzed from queueing theory perspective. More specifically, the problem of storing and transporting perishable items at low cost and minimum wastage is considered by applying the FGBS rule.

\subsubsection{Model Description in Perishable Goods Management.} The following assumptions are made: a bulk of perishable goods arrive at the warehouse according to the Poisson distribution with the composite arrival rate $\lambda$ and they are stored for later shipment with already existing products. The queue of products in the distribution center is served upon arrival of a truck and its capacity is $b$ units. Assume that one truck is used to transport products from one distribution center to customers. The transportation cost and average waiting time will be minimum if always transported with full truckload. Most of products cannot be delivered in this manner, because always the number of items in the distribution center may not be greater than or equal to $b$. In our model, it is assumed that the transportation is performed right after the quantity of goods accumulated in the warehouse reaches:

less than $a$ but the average waiting time of the items is greater than or equal to time $T$, all the items are taken for transport;

lesser than time $T$, the server leaves for a vacation; greater than $a$ but less than $b$, all the items are taken for transport;

more than $b$, first $b$ are taken for transport.

The transportation time follows an exponential distribution with rate $\mu$. If the criterion to transport is not met, the server used to transport the items will take multiple vacations until the system meets the criteria at the end of any vacation. The duration of vacation follows an exponential distribution with rate $\nu$. This type of queue will fall under $M^{X} / M(a, b) / 1$ with flexible general bulk service and multiple vacations. The queue discipline FIFO is suitable for this queue.

5.2.2. Numerical Illustration. The following set of parametric values was considered to simulate the model: The arrival of the batches of perishable items follows the Poisson process with rate $\lambda=1 / 15$ per hr. The size of each such batch is a random variable $X$ following a geometric distribution with probability $p=0.5$. The service time of the truck 
TABLE 7: Performance measures of the system with GBS and FGBS for different values of $a$.

\begin{tabular}{|c|c|c|c|c|c|c|c|c|c|c|}
\hline \multirow{2}{*}{$a$} & \multicolumn{5}{|c|}{ General bulk service rule } & \multicolumn{5}{|c|}{ Flexible general bulk service rule } \\
\hline & $W_{q}$ & $N_{q}$ & $\%$ expired & Utilization & Batch size & $W_{q}$ & $N_{q}$ & $\%$ expired & Utilization & Batch size \\
\hline 17 & 1.3683 & 10.8932 & 0.3788 & 0.3651 & 21.9469 & 1.3731 & 10.9282 & 0.5782 & 0.3644 & 21.5949 \\
\hline 18 & 1.4213 & 11.3470 & 0.6222 & 0.3586 & 22.7400 & 1.4326 & 11.5142 & 0.6408 & 0.3530 & 22.7134 \\
\hline 19 & 1.4697 & 11.6746 & 0.5725 & 0.3301 & 23.5495 & 1.4674 & 11.8292 & 0.7494 & 0.3426 & 23.5003 \\
\hline 20 & 1.5215 & 12.4141 & 0.5487 & 0.3292 & 24.5660 & 1.4970 & 12.0468 & 0.6684 & 0.3383 & 23.9576 \\
\hline 21 & 1.5983 & 12.8005 & 0.9212 & 0.3062 & 25.3675 & 1.4996 & 11.9907 & 0.7771 & 0.4049 & 21.8284 \\
\hline 22 & 1.6962 & 13.4352 & 1.0655 & 0.2998 & 26.1697 & 1.4996 & 12.0074 & 0.6261 & 0.4276 & 20.7278 \\
\hline 23 & 1.7337 & 13.7623 & 1.0501 & 0.2933 & 27.0057 & 1.4998 & 12.0779 & 0.9885 & 0.4825 & 19.3106 \\
\hline 24 & 1.7974 & 14.1976 & 1.1942 & 0.2824 & 27.7859 & 1.5000 & 11.9783 & 0.9021 & 0.5171 & 17.9564 \\
\hline 25 & 1.8373 & 14.8079 & 1.3830 & 0.2787 & 28.5792 & 1.5003 & 12.0026 & 0.7620 & 0.5368 & 17.2707 \\
\hline 26 & 1.9514 & 15.4740 & 1.4379 & 0.2704 & 29.2328 & 1.5016 & 11.8747 & 0.9373 & 0.5793 & 16.2082 \\
\hline 27 & 2.0348 & 16.0935 & 2.1157 & 0.2757 & 29.9008 & 1.5012 & 11.9127 & 1.1121 & 0.5941 & 15.0395 \\
\hline 28 & 2.0845 & 16.5850 & 2.0052 & 0.2604 & 30.4670 & 1.5000 & 11.8125 & 1.2091 & 0.6278 & 14.3389 \\
\hline 29 & 2.1815 & 17.4142 & 2.1735 & 0.2579 & 30.9822 & 1.5005 & 11.9982 & 0.9323 & 0.6289 & 14.1825 \\
\hline 30 & 2.2647 & 17.8726 & 2.3092 & 0.2578 & 31.4699 & 1.5005 & 11.9982 & 1.0188 & 0.6289 & 14.1825 \\
\hline 31 & 2.3896 & 18.9035 & 2.4303 & 0.2448 & 31.8065 & 1.4999 & 12.0510 & 1.3984 & 0.6753 & 13.2010 \\
\hline 32 & 2.4990 & 19.7966 & 3.0774 & 0.2557 & 31.9854 & 1.5035 & 11.8996 & 1.4151 & 0.6855 & 12.6657 \\
\hline
\end{tabular}

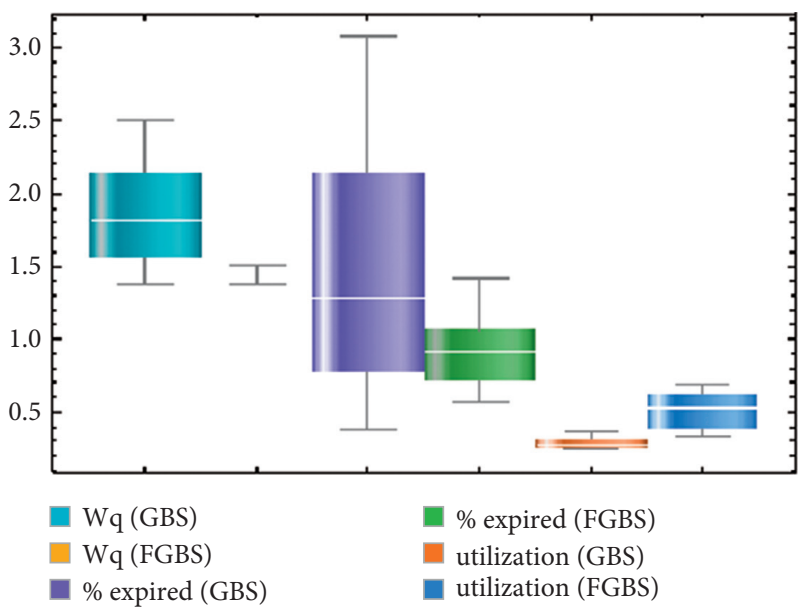

FIGURE 9: Box and whisker plot for comparison.

follows an exponential distribution with rate $\mu=1$ per hr. Maximum truck capacity is $b=32$. The vacation time of the server follows an exponential distribution with rate $v=2$. The lifespan of the product is 7 hrs. Table 7 shows how the FGBS rule is more efficient than the GBS rule for increasing the value of the minimum capacity of truck $a$.

The box and whisker plot shown in Figure 9 was drawn using the values from Table 7 to compare the results from each of the rules. The following is observed:

The average waiting time of a customer is effectively controlled under the FGBS rule. Its minimum value is 1.3731 , maximum value is 1.5035 , median is $1.49995, \mathrm{Q} 1$ is 1.498 3, and Q3 is 1.4995. The box is not visible, since the deviation between Q1 and Q3 has relatively a small value.

Percentage of expiration is very low in case of FGBS rule in comparison to GBS rule. Tolerance of average waiting time of a perishable item in the distribution center $T$ is chosen based on the lifetime of the item.

Utilization of server is large because the frequency of providing service in FGBS is greater than that in GBS.
By applying the FGBS rule in the distribution of perishable items, the risk of spoilage or loss of freshness is minimized. The increase in the utilization of the server may increase the cost but it reduces the cost due to the wastage of items.

\section{Conclusion}

An attempt is made in this article to reduce the waiting time of a customer in the general bulk service queueing system. Flexible general service rule is introduced and analyzed for different $T$ values. It has been proved that the FGBS rule effectively constricts the waiting time of a customer. This model is preeminent for the customer who has a fixed lifespan. Application of the FGBS rule is illustrated in detail with the help of two scenarios, namely, specimen waiting for testing in a clinical laboratory and perishable item waiting for delivery. It is observed that the FGBS rule effectively reduced the expiration of specimen and wastage of perishable items. FGBS rule is a threshold to reduce and control the waiting time of a customer in any type of bulk service. The model can be further extended with restricted admissibility, service and vacation interruptions, and setup time concepts for future research. The proposed queueing model may be studied for discrete time case also. FGBS may be incorporated in a multiple service channel queueing system.

\section{Data Availability}

The data used in the article were taken based on assumption.

\section{Conflicts of Interest}

The authors declare that they have no conflicts of interest.

\section{References}

[1] M. F. Neuts, "A General Class of Bulk Queues with Poisson Input," The Annals of Mathe-matical Statistics, vol. 38, no. 3, pp. 759-770, 1967. 
[2] B. T. Doshi, "Queueing systems with vacations? a survey," Queueing Systems, vol. 1, no. 1, pp. 29-66, 1986.

[3] S. Sasikala and I. Kandaiyan, "Bulk service queueing models-a survey," International Journal of Pure and Applied Mathematics, textbf106, vol. 6, pp. 43-56, 2016.

[4] M. Balasubramanian, R. Arumuganathan, and A. S. Vadivu, "Steady state analysis of a non-Markovian bulk queueing system with overloading and multiple vacations," International Journal of Operational Research, vol. 9, no. 1, pp. 82103, 2010.

[5] S. Jeyakumar and R. Arumuganathan, "Steady state analysis of a non-markovian bulk queue with restricted vacations," International Journal of Operational Research, vol. 10, no. 3, pp. 307-332, 2011.

[6] M. Balasubramanian and R. Arumuganathan, "Steady state analysis of a bulk arrival general bulk service queueing system with modified M-vacation policy and variant arrival rate," International Journal of Operational Research, vol. 11, no. 4, pp. 383-407, 2011.

[7] S. Jeyakumar and B. Senthilnathan, "Steady state analysis of bulk arrival and bulk service queueing model with multiple working vacations," International Journal of Mathematics in Operational Research, vol. 9, no. 3, pp. 375-394, 2016.

[8] R. Arumuganathan and S. Jeyakumar, "A study on bulk arrival non Markovian single service queue with state dependent service," Opsearch, vol. 47, no. 2, pp. 128-142, 2010.

[9] T. Deepa and A. Azhagappan, "Analysis of batch Arrival single and bulk service queue with multiple vacation closedown and repair," Applications and Applied Mathematics, vol. 14, no. 2, pp. 617-639, 2019.

[10] M. Plebani and P. Carraro, "Mistakes in a stat laboratory: types and frequency," Clinical Chemistry, vol. 43, no. 8, pp. 1348-1351, 1997.

[11] M. P. Cornes, J. Atherton, G. Pourmahram et al., "Monitoring and reporting of preanalytical errors in laboratory medicine: the UK situation," Annals of Clinical Biochemistry: International Journal of Laboratory Medicine, SAGE Publications, vol. 53, no. 2, pp. 279-284, 2016.

[12] A. Sakyi, E. Laing, R. Ephraim, O. Asibey, and O. Sadique, "Evaluation of analytical errors in a clinical chemistry laboratory: a 3 year experience," Annals of Medical and Health Sciences Research, vol. 5, no. 1, pp. 8-12, 2015.

[13] D. Najat, "Prevalence of pre-analytical errors in clinical chemistry diagnostic labs in sulaimani city of Iraqi Kurdistan," PLoS One, vol. 12, Article ID e0170211, 2017. 